\title{
Studies on the robustness of shock-ignited laser fusion targets
}

\author{
$\underline{\text { S. Atzeni }}^{1}$, A. Schiavi ${ }^{1}$, A. Marocchino ${ }^{2}$, A. Giannini ${ }^{2}$ \\ ${ }^{1}$ Dipartimento di Energetica, Università di Roma "La Sapienza” and CNISM, Italy \\ ${ }^{2}$ Dipartimento di Energetica, Università di Roma "La Sapienza”, Italy
}

\section{Introduction}

Shock ignition, SI, [1] is a promising approach to inertial confinement fusion (ICF). Like fast ignition, it separates the stages of compression and ignition, but does not require ultraintense lasers, and may allow for relatively simple spherical targets. Recently, it has been shown that the HiPER baseline target [2], originally conceived for fast ignition, can be shock-ignited [3] and that shock ignition could be tested at full scale on the National Ignition Facility (NIF) [4].

We therefore deem the study of the robustness of SI targets worthy and timely. Here, we present results of a numerical study concerning both the compression phase and ignition and gain. Simulations were performed with the lagrangian code DUED [5], using either its 1D version or the full $2 \mathrm{D}$ version. The whole target evolution was studied with the complete code physical model, including multigroup radiation diffusion, alpha-particle diffusion, 2D laser raytracing. A parametric scan of the compression phase (requiring more than 10000 simulations) was instead performed with a simpler model, assuming radial laser propagation, and neglecting radiative transfer.

We mainly refer to the HiPER baseline target [2], and also consider a target proposed by the CELIA group [6] in May 2010 for testing the concept at NIF. The first one consists in a simple DT shell with outer radius of $1.044 \mathrm{~mm}$ and thickness of $211 \mu \mathrm{m}$. The second one has outer radius of $898 \mu \mathrm{m}$, with an outer $\mathrm{CH}$ layer of $28 \mu \mathrm{m}$ and a $200 \mu \mathrm{m}$ thick inner DT layer. Both targets are filled with DT vapour at density of $0.1 \mathrm{mg} / \mathrm{cm}^{3}$. These targets are irradiated by laser pulses with wavewlength $\lambda_{\text {laser }}=0.35 \mu \mathrm{m}$ and power shaped in time as shown in Fig. 1 .

\section{Compression stage}

1D compression performance, measured by the peak value of the confinement parameter $<\rho R>=\int \rho d r,(\rho$ : density; $r$ radial variable) depends on a few parametres defining the target dimensions and mass, and laser power evolution. We have performed a large scan by varying simultaneously the total laser energy, the DT density and the DT shell radii of the HiPER baseline case. Complete results will be presented elsewhere. Figure 2 shows the effects of varying one parameter at a time around the nominal value. We see that limiting $\langle\rho R>$ relative deviations from the nominal value to $\pm 10 \%$ requires limiting the changes of radii to less than $2 \%$, while 

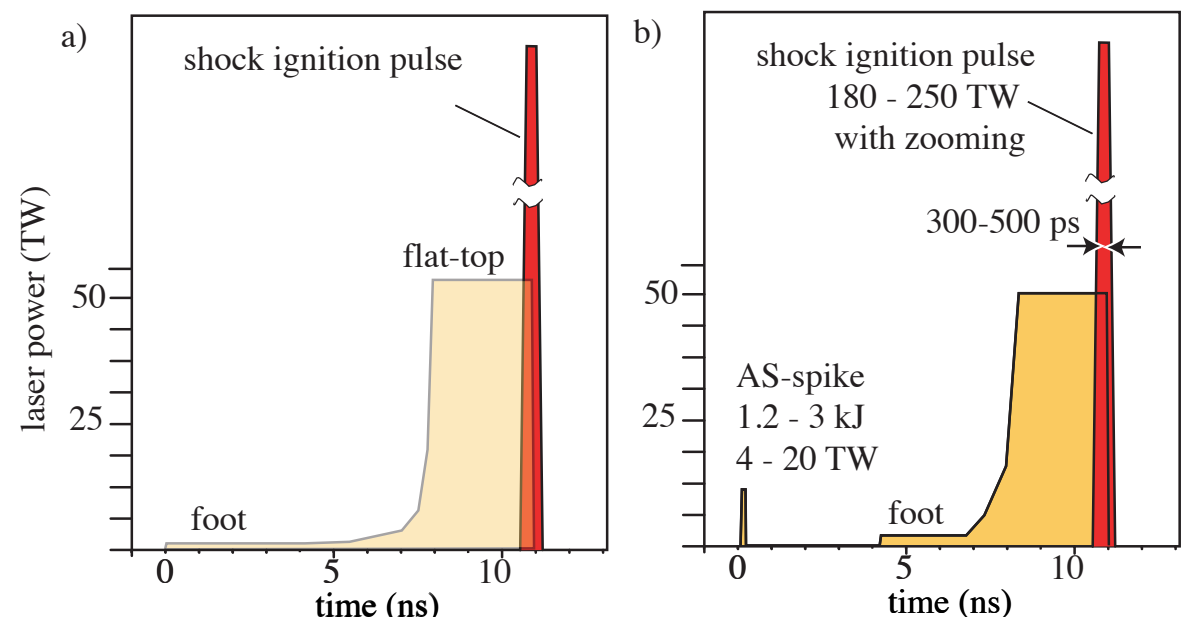

Figure 1: Laser power vs time. a) standard pulse; b) pulse with adiabat-shaping (AS) picket. The orange-filled part is the compression pulse; the red-filled one is the shock ignition pulse, which adds to the compression pulse. The numbers in the figures refer to the HiPER baseline target, irradiated by cylindrical beams (see text).

density and energy variations can be as large as $6-7 \%$.

The CELIA group studied the asymmetry of the compressed fuel caused by the irradiation nonuniformities associated to a nominal irradiation geometry [7]. They found relatively large deformations of the compressed fuel and of the central hot spot. However, they also showed that this target can be shock-ignited [3]. Actually, shock collision and ignition limit the growth of RTI at the inner shell target. We recovered this result. We have extended this study by addressing the tolerance to target positioning via $2 \mathrm{D}$ simulations. Cylindrical symmetry was assumed and the target was displaced along the symmetry axis with respect to the nominal target chamber centre. As in Ref. [7], we assumed radial laser rays and laser intensity perturbation given as a sum of Legendre modes $A_{l} P_{l}(\cos (\theta))(\theta$ : polar angle). The nominal irradiation geometry is considered again. It is found that the mode $l=1$ grows linearly with the relative displacement. The other modes are not significantly affected below the $1 \%$ displacement threshold, but the amplitude of satellite modes becomes significant and comparable to the initial modes for a displacement in the range of 2 to $5 \%$, ie for a 20 to $50 \mu \mathrm{m}$ offset. The tolerance of the target has been investigated for $0 \%, 1 \%$ and $5 \%$ relative displacement. We used the areal density $\langle\rho R>$ averaged over the polar angle and the rms deviation of $\langle\rho R\rangle(\theta)$ as figures of merit of the implosion. We find (see Fig. 3) that the $1 \%$ and 5\% displacement runs present the same mean $\rho R$ as the reference run. On the other hand, the rms deviations from the mean (drawn as banded coloured regions in Fig. 3) become more and more significant with increasing displacement. 


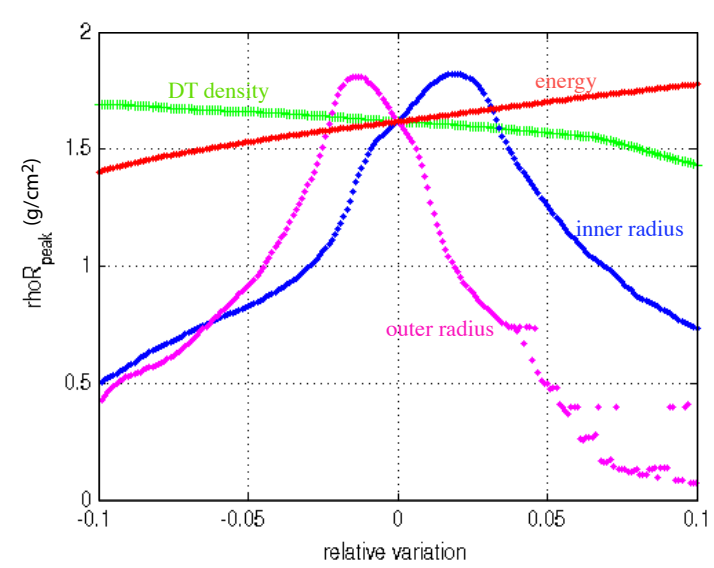

Figure 2: Peak confinement parameter vs relative variation of four parameters.

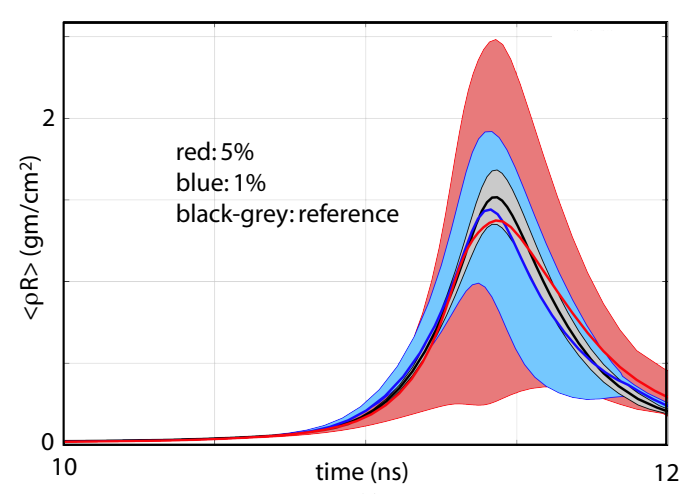

Figure 3: Curves: Average $\rho R$ vs time for $0 \%, 1 \%$ and $5 \%$ relative displacement of target centre. Coloured regions: see text.

We also studied the evolution of single wavelength, small-scale, small-amplitude, perturbations of the outer surface of the target [10]. This is subjected to Richtmyer-Meshkov Instability, RMI, [9] during the foot of the pulse, and then to the more violent RTI, during the final portion of the ramp and the flat-top. An important result is that RMI is nearly suppressed and RTI growth rate is significantly reduced for modes $l>300$, when the pulse is preceeded by an adiabat shaping (AS) picket [8]; see Fig. 4.
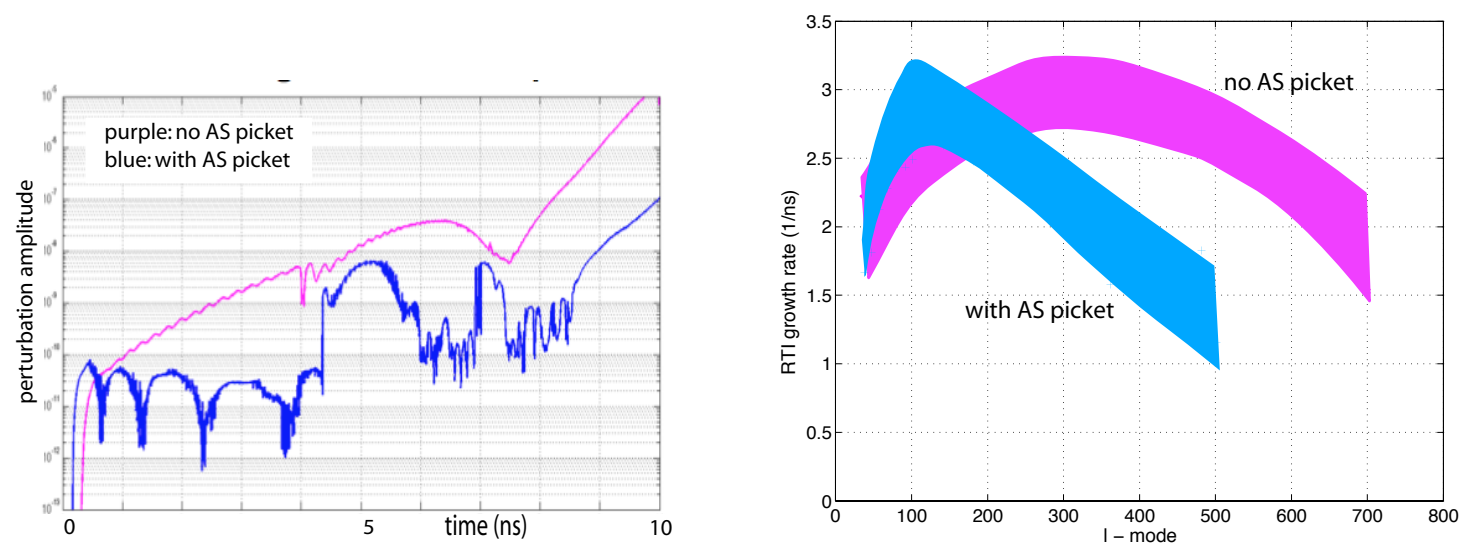

Figure 4: Left: evolution of ablation front perturbations with $l=300$; purple: pulse without picket; blue: pulse with picket. Right: RTI growth rates vs mode number $l$, in the interval 7.5 $9.5 \mathrm{~ns}$. 


\section{Ignition and gain robustness}

We then studied ignition of the HiPER baseline target driven by pulses preceeded by an adiabat-shaping picket, which was not considered in the CELIA study. By appropriately shaping the pulse we recover CELIA results [3], concerning power threshold for ignition, pulse synchronization, and gain windows. For ideal radial irradiation the mininum power of the SI pulse is about $60 \mathrm{TW}$ (to be added to $26 \mathrm{TW}$ of the compression pulse). An optimal power (maximising gain and corresponding to a relatively large time window) is about $80 \mathrm{TW}$. However, this result changes substantially when more realistic irradiation geometry is considered. Assuming cylindrical beams, with intensity profile $\exp (-r / w)^{m}$ with $m=2$ and $w=0.64 \mathrm{~mm}$ (optimal for the initial stage of compression) the SI beam is absorbed very poorly. When the width of the SI beams is reduced to $0.4 \mathrm{~mm}$ ignition requires compression beam power of 42-50 TW and SI beam power in excess of $190 \mathrm{TW}$, with an optimal value probably around $220 \mathrm{TW}$. At $220 \mathrm{TW}$ the minimum SI spike FWHM duration is about 400 ps. The maximum 1D gain is close to 80 .

The target proposed by Schurtz and Ribeyre [6], is irradiated by superGaussian beams with $m=4$ and $w=683 \mu \mathrm{m}$ for the compression stage and $w=345 \mu \mathrm{m}$ for the SI pulse. Ignition requires compression energy of about $250 \mathrm{~kJ}$ (with peak power of $80 \mathrm{TW}$ ) and SI pulse power exceeding $150 \mathrm{TW}$ and energy exceeding $70 \mathrm{KJ}$. The target releases about $33 \mathrm{MJ}$ of fusion energy with gain 80-100. The hot-spot convergence ratio at ignition time is quite high, $C_{\mathrm{h}}=$ 43 , for DT vapour density of $0.1 \mathrm{mg} / \mathrm{cm}^{3}$. However, we have found that such density can be increased to $0.5 \mathrm{mg} / \mathrm{cm}^{3}$ with a minor reduction of the $1 \mathrm{D}$ gain; correspondingly $C_{\mathrm{h}}$ is reduced to about 30 . This is a promising result, which however has to be confirmed by 2D simulations.

We thank G. Schurtz and J. Ribeyre for providing us results prior to publication. Work partially supported by the Italian MIUR project PRIN 20072KW45J and by HiPER project and Preparatory Phase Funding Agencies (EC, MSMT and STFC).

\section{References}

[1] R. Betti et al., Phys. Rev. Lett. 98, 155001 (2007).

[2] S. Atzeni, A. Schiavi and C. Bellei, Phys. Plasmas 14, 052702 (2007).

[3] X. Ribeyre et al., Plasma Phys. Controll. Fusion 51015013 (2009).

[4] L. J. Perkins et al., Phys. Rev. Lett. 103, 045004 (2009).

[5] S. Atzeni et al., Computer Phys. Commun. 169, 153 (2005).

[6] G. Schurtz and J. Ribeyre, private communication (May 2010).

[7] L. Hallo et al., Plasma Phys. Controll. Fusion 51, 014001 (2009).

[8] K. Anderson and R. Betti, Phys. Plasmas 11, 5 (2004).

[9] V. N. Goncharov et al., Phys. Plasmas, 13, 012702 (2006).

[10] A. Marocchino, S. Atzeni, A. Schiavi, these proceedings, paper P4.223. 